\title{
Topological Phase Transition in a One-Dimensional Elastic String System
}

\author{
Ya-Wen Tsai ${ }^{1}{ }^{\mathbb{D}}$, Yao-Ting Wang ${ }^{2}$, Pi-Gang Luan ${ }^{3, *}$ and Ta-Jen Yen ${ }^{1, *}$ \\ 1 Department of Materials Science and Engineering, National Tsing Hua University, Hsinchu 30013, Taiwan; \\ s106031851@m106.nthu.edu.tw \\ 2 Department of Physics, Imperial College London, London SW7 2AZ, UK; ywang14@ic.ac.uk \\ 3 Department of Optics and Photonics, National Central University, Jhongli 32001, Taiwan \\ * Correspondence: pgluan@dop.ncu.edu.tw (P.-G.L.); tjyen@mx.nthu.edu.tw (T.-J.Y.)
}

Received: 20 May 2019; Accepted: 15 June 2019; Published: 18 June 2019

\begin{abstract}
We show that topological interface mode can emerge in a one-dimensional elastic string system which consists of two periodic strings with different band topologies. To verify their topological features, Zak-phase of each band is calculated and reveals the condition of topological phase transition accordingly. Apart from that, the transmittance spectrum illustrates that topological interface mode arises when two topologically distinct structures are connected. The vibration profile further exhibits the non-trivial interface mode in the domain wall between two periodic string composites.
\end{abstract}

Keywords: Zak phase; topological interface states; string waves; 1D crystals

\section{Introduction}

The recent discovery of topological features in condensed matter systems [1-8], for example, chiral edge states [9-11] or effective magnetic fields [12] have attracted massive interests [13,14]. The topological phases in electronic systems can be characterized by the band topologies and the sum over their corresponding topological invariants below the Fermi level $[12,15,16]$. Among all the topological materials, a one-dimensional (1D) polyethylene chain governed by Su-Schrieffer-Heeger (SSH) model with staggered hopping parameters $[17,18]$ is a well-studied example to exhibit topological nature. To study the band topology in a 1D periodic system with inversion symmetry, the Zak phase is introduced to facilitate the topological classification [2,19]. Furthermore, when two SSH chains with distinct staggered hopping are connected, interface states arise at the domain wall as long as the corresponding Zak phases for both subdomains are different. Recent researches have demonstrated that the Zak phase also relates to the reflection phase and surface impedance $[20,21]$.

In a similar manner, the topological aspects can apply to classical systems. Several topological properties have been predicted and observed in photonic crystals, coupled with resonators and linear circuits [22-28]. While the research to topological phenomena has also been investigated in various classical wave models [29-31], topological properties of pre-tensioned strings remain unnoticed. Since the vibrating frequency and amplitude of a pre-tensioned string system can be easily measured, the system shows great potential to directly observe topological properties. The purpose of the study is to explore the topological phase transition in a 1D elastic string (ES) system through the analysis of the related topological interface mode. The main aims in this report are to identify the topological quantities in the band structure and to elucidate the relation between the interface mode and the band topologies of the two 1D periodic ES subsystems (See Figure 1). 


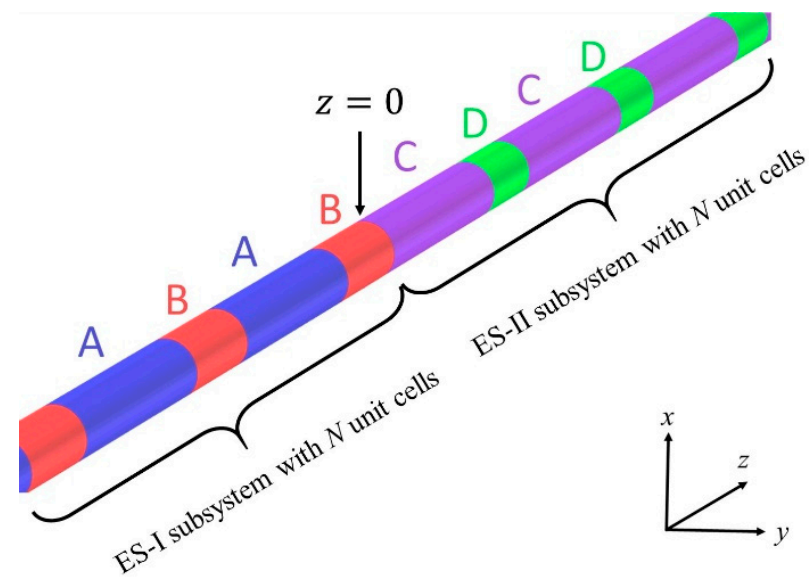

Figure 1. A one-dimensional elastic string system composed of ES-I and ES-II subsystems at the interface $(z=0)$.

\section{A One-Dimensional Periodic Elastic String}

\subsection{Dispersion Relation}

We begin by considering the wave equation of a 1D periodic ES in which the internal stress of the string is omitted. Assuming that the entire structure is stretched by an external force to provide the tension, along the z-axis, we can write down the Lagrangian density for the string wave.

$$
L=\frac{\rho}{2}\left(\partial_{t} u\right)^{2}-\frac{\tau}{2}\left(\partial_{z} u\right)^{2},
$$

where $u, \tau$ and $\rho$ are the transverse displacement, the string tension, and the linear density of the string, respectively. According to the Euler-Lagrange equation, the wave equation is therefore given by

$$
\partial_{z}\left(\tau \partial_{z} u\right)=\rho \partial_{t}^{2} u
$$

Due to the periodicity of density $\rho$, a Bloch theorem $u=e^{-i q z} u_{q}(z)$ is introduced with the time convention $e^{i \omega t}$, where $u_{q}(z)=u_{q}(z \pm \ell)$ is the periodic Bloch wave, $q$ is Bloch wave vector, and $\ell$ is lattice constant. The unit cell contains two homogeneous string segments with lengths $\ell_{1}$ and $\ell_{2}$, and their densities are $\rho_{1}$ and $\rho_{2}$. By imposing the boundary conditions $u$ and $\partial u / \partial z$ at the segment-segment interfaces, one can derive the transfer matrix $T$ for one-unit cell. Since the transverse displacement in the first string segment (with length $\ell_{1}$ ) of the $j$ th unit cell is expressed as $u_{j}(z)=a_{j} e^{-i k_{1}(z-j \ell)}+b_{j} e^{i k_{1}(z-j \ell)}$, via periodic boundary condition $u(z+\ell)=e^{-i q \ell} u(z)$, the matrix equation reads [32]:

$$
\left(\begin{array}{ll}
T_{11} & T_{12} \\
T_{21} & T_{22}
\end{array}\right)\left(\begin{array}{c}
a_{j} \\
b_{j}
\end{array}\right)=e^{i q \ell}\left(\begin{array}{c}
a_{j} \\
b_{j}
\end{array}\right)
$$

where the matrix elements $T_{11}, T_{12}, T_{21}$, and $T_{22}$ can be found in Ref. [32]. Determined by the condition $\operatorname{det}\left(T-e^{i q \ell} I\right)=0$, the dispersion relation yields

$$
\cos q \ell=\cos k_{1} \ell_{1} \cos k_{2} \ell_{2}-\frac{1}{2}\left(\frac{k_{2}}{k_{1}}+\frac{k_{1}}{k_{2}}\right) \sin k_{1} \ell_{1} \sin k_{2} \ell_{2}
$$

where $k_{\alpha}=\omega / v_{\alpha}, v_{\alpha}=\sqrt{\tau / \rho_{\alpha}}$, and $\alpha=1$ or 2 . Assuming that the parameters of tension and materials are $\tau=477.46 \mathrm{~Pa}, \rho_{A}=2704 \mathrm{~kg} / \mathrm{m}^{3}, \rho_{B}=8100 \mathrm{~kg} / \mathrm{m}^{3}, \ell_{A}=0.6759 \ell$ and $\ell_{B}=0.3241 \ell$, the result of the band structure is shown in Figure 2. 


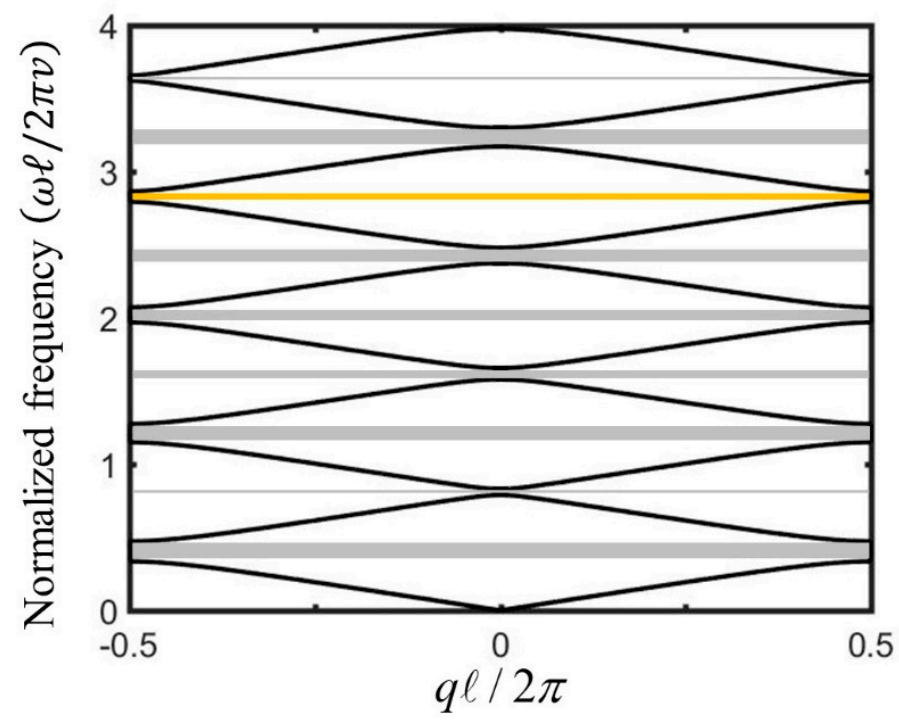

Figure 2. The band structure (solid black curves) with the parameters $\rho_{A}=2704 \mathrm{~kg} / \mathrm{m}^{3}, \rho_{B}=8100$ $\mathrm{kg} / \mathrm{m}^{3}, \ell_{A}=0.6759 \ell$ and $\ell_{B}=0.3241 \ell$. Grey and orange strips represent the band gaps. The topological properties of the orange strip are considered in our main text.

\subsection{Band Crossing Condition}

As mentioned, the Zak phase plays an important role in the classification of topological insulators, and the interface mode arises around the domain wall of two topologically distinct band structures [21, 33]. When we continuously tune the density of the B-segment $\rho_{B}$ from $8100 \mathrm{~kg} / \mathrm{m}^{3}$ to $9580 \mathrm{~kg} / \mathrm{m}^{3}$ as well as varying the segment lengths $\ell_{A}$ and $\ell_{B}$ in one-unit cell, the result shows that the gap reopens as shown in Figure 3. Meanwhile, following the condition (see Appendix A)

$$
\frac{k_{A} \ell_{A}}{k_{B} \ell_{B}}=\frac{m_{1}}{m_{2}}
$$

where $m_{1}$ and $m_{2}$ are two co-prime integers, the band crossing occurs within the $\left(m_{1}+m_{2}\right)$ th band-gap at Brillouin center and boundary $( \pm \pi / q \ell)$. In the same manner, our design shows that the band inversion occurs around the 7th bandgap. In the subsequent content, we will focus on the band structure around this band.

(a)

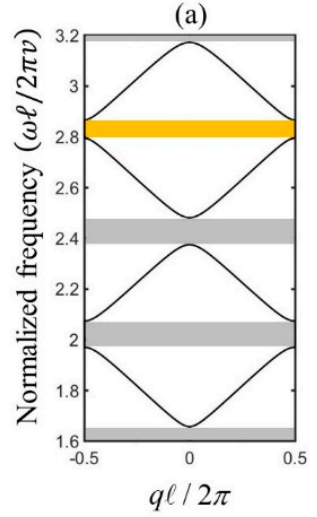

(b)

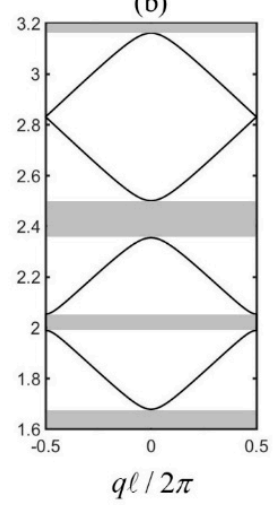

(c)

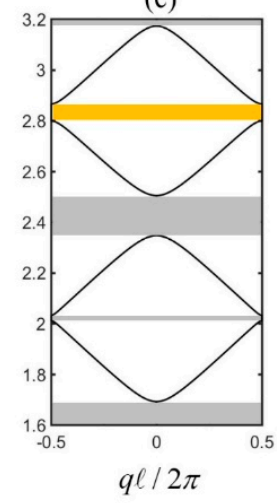

Figure 3. (a-c) are the band structures (solid black curves) of ES-I, a band-crossing ES system, and ES-II, respectively. The material parameters are listed as follows: $\rho_{A}=2704 \mathrm{~kg} / \mathrm{m}^{3}, \rho_{B}=8100 \mathrm{~kg} / \mathrm{m}^{3}$, $\ell_{A}=0.6759 \ell$ and $\ell_{B}=0.3241 \ell ; \rho_{1, \text { band crossing }}=2704 \mathrm{~kg} / \mathrm{m}^{3}, \rho_{2, \text { band crossing }}=8836 \mathrm{~kg} / \mathrm{m}^{3}, \ell_{1, \text { band crossing }}=$ $0.7068 \ell$ and $\ell_{2, \text { band crossing }}=0.2932 \ell ; \rho_{C}=2704 \mathrm{~kg} / \mathrm{m}^{3}, \rho_{D}=9580 \mathrm{~kg} / \mathrm{m}^{3}, \ell_{C}=0.7316 \ell$ and $\ell_{D}=0.2684 \ell$. The gap where the band inversion occurs is highlighted in orange. 


\subsection{Zak Phase}

To classify the topological bands of ES-I and ES-II systems, the Zak phase of each band in both systems are evaluated. The Zak phase for $n$th band is given by [19].

$$
\Theta_{n}^{Z a k}=\int_{-\pi / \ell}^{\pi / \ell} A_{n}^{Z a k}(q) \cdot d q
$$

where $A_{n}^{Z a k}(q)=i \int_{\text {unit cell }} \rho(z) u_{n, q}^{*}(z) \partial_{q} u_{n, q}(z) d z$ is Zak connection. In general, Zak connection is not globally defined in the whole $q$-space owing to the existence of a singularity. If a discontinuous point of Zak connection exists in $q$-space, a gauge transformation must be introduced to make Zak connection evolution analytic. With regard to inversion symmetry, the value of the Zak phase arises from the singularity within Zak connection evolution. Figure 4 shows the evolution of Zak connections for the 7th and 8th bands of the ES-I and the ES-II systems. As shown in Figure 4b,c, Zak phase vanishes when its evolution is continuous. Yet, if the evolution curve contains a singularity, to smoothen the evolution of $u_{q}(z)$, Zak phase gives a value of $\pi$ (see Appendix B). As a result, the singularities appearing in Figure $4 \mathrm{a}$,d imply that the corresponding band has a topological phase $\pi$, showing the emergence of topological phase transition in the ES system. Moreover, $u_{q}(z)$ can be visualized as the " $2 \mathrm{D}$ field" which is the distribution of vibration mode. This provides an alternative method for comparing the difference of topologies between ES-I and ES-II, as depicted in Figure 5. Figure 5b,c present the field continuity in momentum space. In Figure $5 \mathrm{a}, \mathrm{d}$, however, the vibration modes show a discontinuous nature consistent with the locations of the singularities in Figure 4 . Therefore, it must assign a minus sign to the vibration mode while $q$ evolves across the singularity point. As the inversion symmetry holds, this sign change from this gauge transformation will give an extra phase $\pi$.

(a)

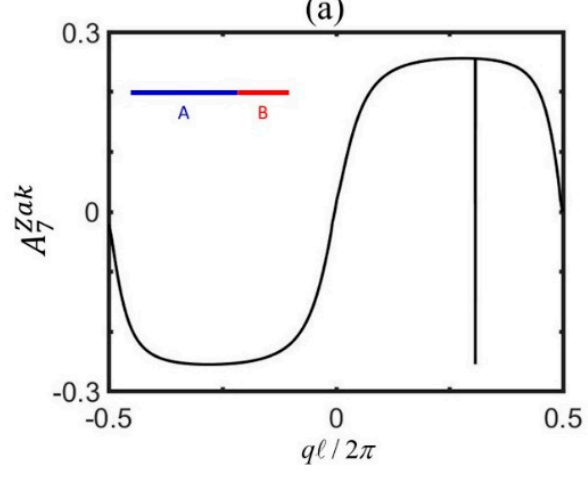

(c)

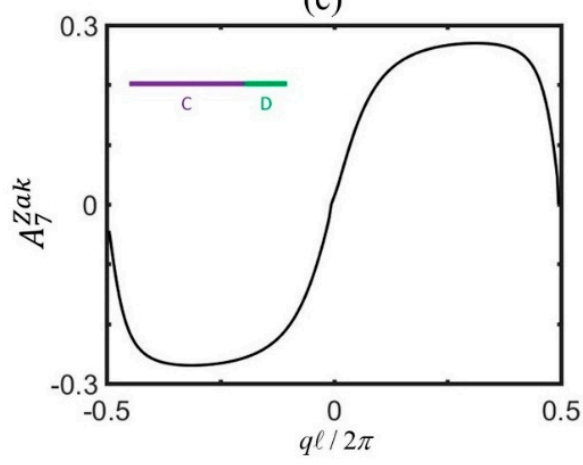

(b)

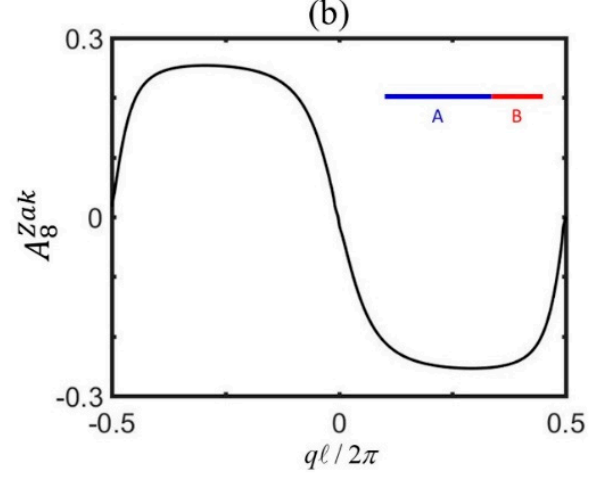

(d)

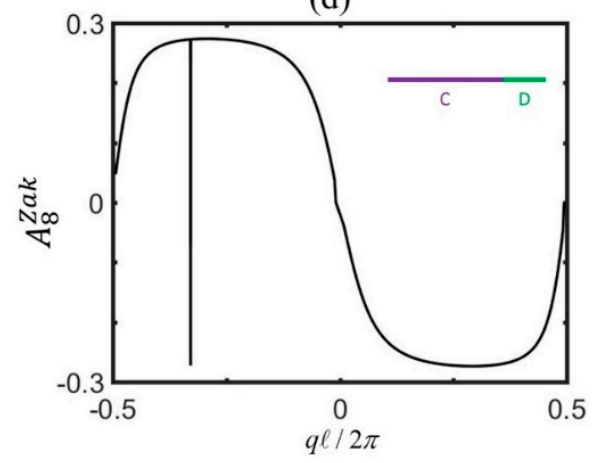

Figure 4. The evolution of Zak connections for the 7th and 8th bands of the ES-I and the ES-II. $(\mathbf{a}, \mathbf{b})$ are the results of the 7th and 8th band of ES-I. A singularity of Zak connection appears in (a) within Brillouin zone. (c,d) are the results for the 7th and 8th band of ES-II. After the band inversion, the Zak connection evolution shows that the singularity in 7th band (a) band has switched to the 8th band (d). Due to the gauge transformation, the two position of the singularities in $(\mathbf{a}, \mathbf{d})$ are symmetric. 
(a) Band $7^{\text {th }}($ ES-I)

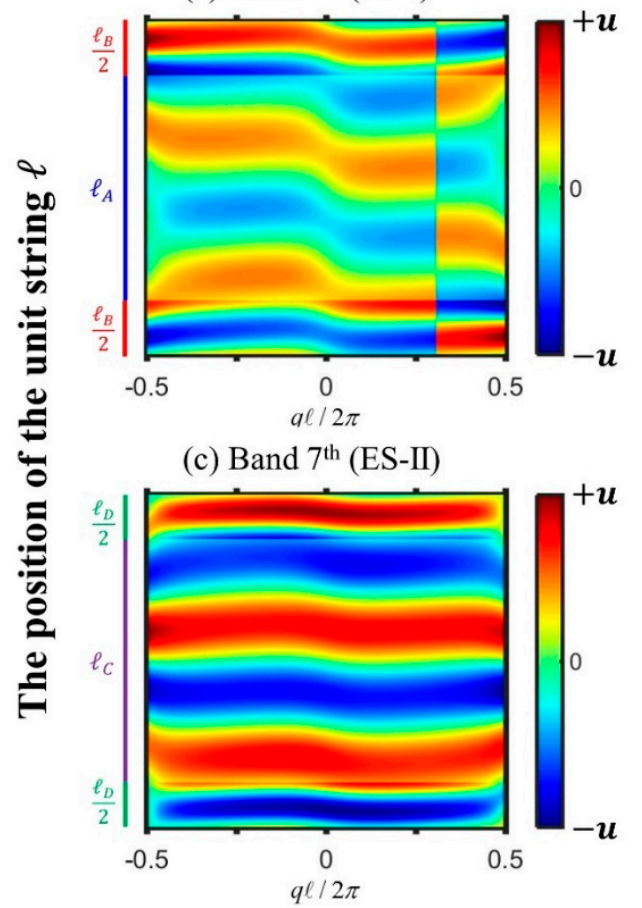

(b) Band $8^{\text {th }}($ ES-I)

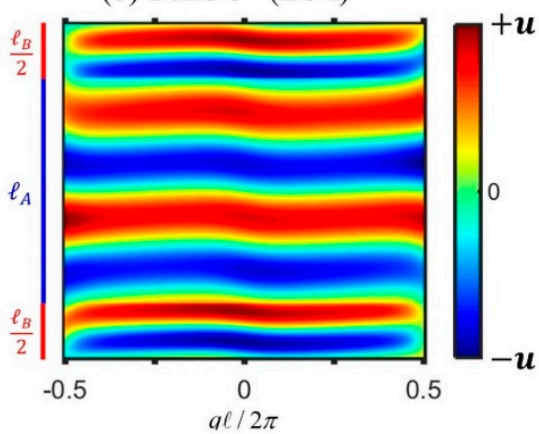

(d) Band $8^{\text {th }}$ (ES-II)

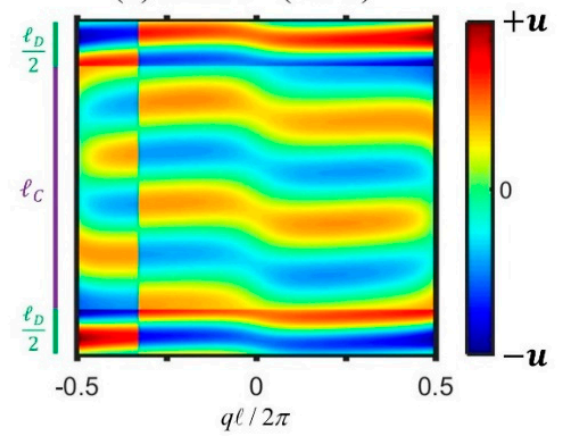

Figure 5. The four panels show the vibration modes of ES-I and ES-II in $q$ space. The results for the 7th and 8th bands of ES-I are shown in $(\mathbf{a}, \mathbf{b})$. The results for the 7 th and 8th bands of ES-II are shown in $(\mathbf{c}, \mathbf{d})$. The two discontinuities in $(\mathbf{a}, \mathbf{d})$ correspond to the singularities in the Zak connection evolution (Figure 4).

\section{The Interface Mode}

To verify the emergence of the interface mode, a 1D composite ES system consisting of 20 unit cells of ES-I and ES-II is discussed. The corresponding band structures of the ES-I and the ES-II systems are demonstrated in Figure 6a,b. Although the wave propagation within a bandgap is forbidden, the interface state appears as a sharp peak in the transmission spectrum. Figure $6 \mathrm{c}$ illustrates that the common gap (the 7th gap) of the ES-I and ES-II supports this topological interface mode at the frequency 2.83, which matches the location of the peak in the transmission spectrum. This interface mode can also be examined by investigating the vibrating amplitude $u(z)$ with the frequency of a source 2.82 on the left end of the composite string as shown in Figure 7. Yet, due to the finite size effect and the limitation of numerical accuracy, the peak in Figure $6 \mathrm{c}$ and the corresponding interface resonance in Figure 7 have acceptable inaccuracy less than 1\%. The amplitude distribution of the vibrating string in Figure 7 is also consistent with the bulk-edge correspondence principle, which says that interface mode or edge mode exists at the interface between two regions with distinct band topologies. 
(a)

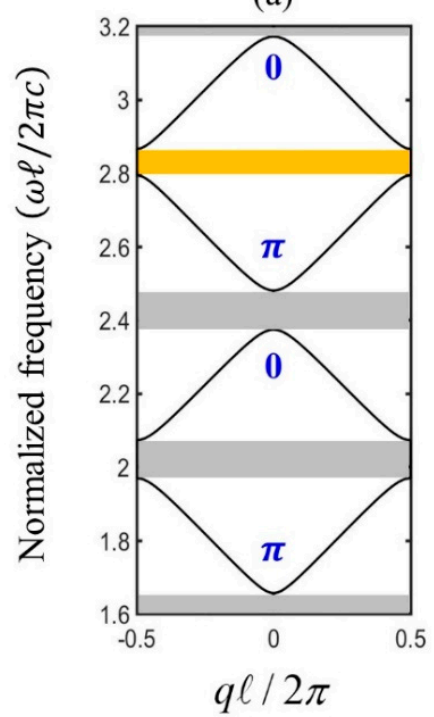

(b)

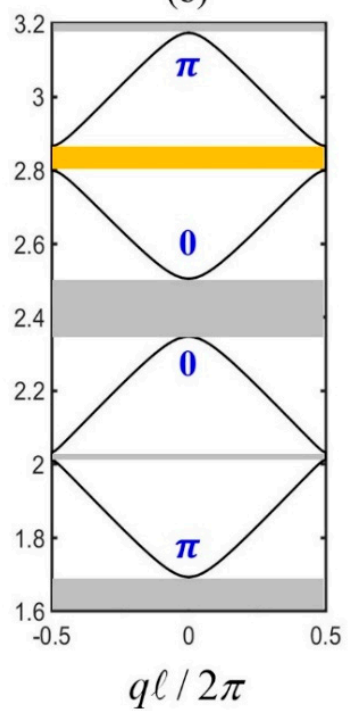

(c)

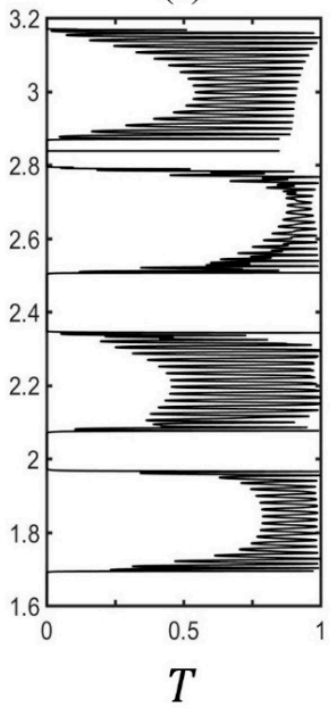

Figure 6. $(\mathbf{a}, \mathbf{b})$ are the band structures (black solid curves) of ES-I and ES-II. The band inversion occurs at orange strips. Zak phase (blue number) of each band is also marked at the center of the band in $(\mathbf{a}, \mathbf{b})$. (c) The transmission spectrum of a composite ES system composed of 20-unit cells on both sides of the interface. The sharp peak at 2.83 indicates the interface mode.

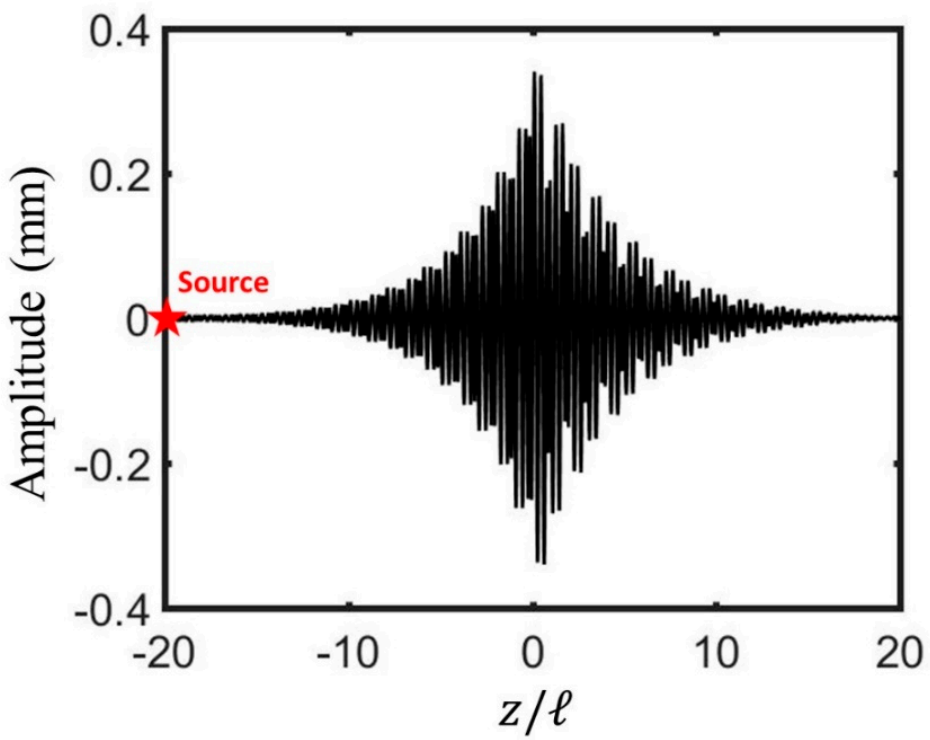

Figure 7. The interface mode of the ES-I/ES-II composite system can be excited by dimensionless frequency 2.82 .

\section{Possible Experimental Implementation}

Since the proposed 1D ES system is made by aluminum, $\mathrm{Cu}-\mathrm{Zn}$ alloy, and $\mathrm{Cu}-\mathrm{Pb}$ alloy, it is feasible to experimentally observe the topological interface mode. For the topological interface mode excitation, vibration source can be set by a function generator connected to a commercial String Vibrator named PASCO WA-9857A [34]. Additionally, as a small amplitude of vibration waves can avoid metal fatigue, the interface mode may be excited without structure damage. In our design, the operating frequency of 2.82 corresponds to an ES lattice constant of about $1 \mathrm{~cm}$, which is feasible to fabricate by using current alloy-wire fabrication technology. 


\section{Conclusions}

In conclusion, we have theoretically studied the topological properties of a 1D composite ES system and reveal the topological phase transition arises from band crossing. The Zak connection evolutions of the corresponding periodic subsystems with and without the occurrence of the topological phase transition have been investigated. Moreover, the patterns of the ES vibration modes of the subsystems in $q$ space further confirm the topological characteristics of the subsystems. Therefore, the interface mode emerges as indicated by the sharp peak in the transmission spectrum when both bandgaps align with subsystems and have different topologies. Finally, a viable way for future experimental demonstration is discussed.

Author Contributions: Conceptualization and methodology, P.-G.L. and Y.T.W.; investigation, Y.W.T.; writing—original draft preparation, Y.W.T.; writing—review and editing, T.-J.Y., P.-G.L. and Y.T.W.; Supervisor, T.-J.Y.

Funding: This research was funded by Leverhulme Trust (Grant No. RPG-2016-365) and Ministry of Science and Technology (Grant Nos. 107-2923-E-007-006-MY2, 107-2221-E-007-016-MY3, and 106-2221-E-007 -038 -MY3).

Acknowledgments: The authors would like to thank the reviewers for their nice comments and suggestions to improve the quality of the paper.

Conflicts of Interest: The authors declare no conflict of interest.

\section{Appendix A. Band Crossing Condition}

In this appendix, we prove that the band crossing condition given in Equation 4 (main text). Since there is no absorption in each segment string, the passband satisfies the condition that the absolute value of the r.h.s. of Equation 4 (main text) is smaller than or equal to 1 . When we define $\gamma \equiv k_{A} \ell_{A}+$ $k_{B} \ell_{B}$ into Equation 4 (main text), it can be rewritten as

$$
\cos q \ell=\cos \gamma-\kappa \sin k_{A} \ell_{A} \sin k_{B} \ell_{B}
$$

where $\gamma$ is the phase delay in a unit cell, $\kappa \equiv-1+\left(k_{B} / k_{A}+k_{A} / k_{B}\right) / 2$, and $\kappa>0$. If $\gamma=m \pi$, the absolute value of the r.h.s. of Equation (A1) is larger than 1 on the two conditions: (i) $\cos \gamma=-1$ and $\sin k_{A} \ell_{A}$ $\sin k_{B} \ell_{B} \geq 0$ or (ii) $\cos \gamma=1$ and $\sin k_{A} \ell_{A} \sin k_{B} \ell_{B} \leq 0$. Thus, the frequency must locate in the gap if the two bands do not cross. Nevertheless, if the two bands cross at $\gamma=m \pi$, both of $\sin k_{A} \ell_{A}$ and $\sin k_{B} \ell_{B}$ equal to 0 so that Equation 5 in the main text can be derived.

Moreover, when $\sin \left(k_{A} \ell_{A}\right)=0, \sin \left(k_{B} \ell_{B}\right)=0, \cos \left(k_{A} \ell_{A}\right)=(-1)^{m 1}$ and $\cos \left(k_{B} \ell_{B}\right)=(-1)^{m 2}$, we have $\cos (q \ell)=(-1)^{m 1+m 2}$. Thus, $q=0$ when $m_{1}+m_{2}$ is even, and $q= \pm \pi / \ell$ when $m_{1}+m_{2}$ is odd. In the main text, the parameters of the band crossing system are listed in Figure 3, which gives

$$
\frac{k_{1, \text { band crossing }} \ell_{1, \text { band crossing }}}{k_{2, \text { band crossing }} \ell_{2, \text { band crossing }}}=\frac{\sqrt{\rho_{1, \text { band crossing }}} \ell_{1, \text { band crossing }}}{\sqrt{\rho_{2, \text { band crossing }}} \ell_{2, \text { band crossing }}}=\frac{4}{3} .
$$

As shown in Figure 3b, with $m_{1}+m_{2}=7$ the band crossing occurs within the 7th band gap at Brillouin boundary $\pm \pi / q \ell$.

\section{Appendix B. Zak Phase Calculation for Each Band}

With inversion symmetry, we prove that if Zak connection evolution for an isolated band exists a singularity, then the Zak phase of this band must be $\pi$. Assuming that an open curve exists in the parameter space, all the components of $|u(\mathbf{R})\rangle$ on the curve are smooth. Firstly, we consider the relative phase between two neighboring states on the open curve. The relative phase factor corresponding to the two parameters $\mathbf{R}$ and $\mathbf{R}+d \mathbf{R}$ is

$$
e^{-i \Delta \Theta}=\langle u(\mathbf{R}) \mid u(\mathbf{R}+d \mathbf{R})\rangle .
$$


Thus, the phase difference $\Delta \Theta$ is therefore given by

$$
\Delta \Theta=-\operatorname{Im}[\ln \langle u(\mathbf{R}) \mid u(\mathbf{R}+d \mathbf{R})\rangle] .
$$

When $d \mathbf{R} \rightarrow 0$, to the first-order expansion Equation (A3) becomes,

$$
\begin{aligned}
1-i \Delta \Theta & =\lim _{d \mathbf{R} \rightarrow 0}\left\{\langle u(\mathbf{R}) \mid u(\mathbf{R})\rangle+\left\langle u(\mathbf{R}) \mid \nabla_{\mathbf{R}} u(\mathbf{R})\right\rangle \cdot d \mathbf{R}\right\} \\
& =\lim _{d \mathbf{R} \rightarrow 0}\left\{1+\left\langle u(\mathbf{R}) \mid \nabla_{\mathbf{R}} u(\mathbf{R})\right\rangle \cdot d \mathbf{R}\right\}
\end{aligned}
$$

Thereby, the phase difference $\Delta \Theta$ is

$$
\Delta \Theta=-\operatorname{Im}\left\langle u(\mathbf{R}) \mid \nabla_{\mathbf{R}} u(\mathbf{R})\right\rangle \cdot d \mathbf{R},
$$

where $-\operatorname{Im}\left\langle u(\mathbf{R}) \mid \nabla_{\mathbf{R}} u(\mathbf{R})\right\rangle=i\left\langle u(\mathbf{R}) \mid \nabla_{\mathbf{R}} u(\mathbf{R})\right\rangle$ is Berry connection. For a closed loop, Zak phase $\Theta$ is expressed as

$$
\Theta=-\operatorname{Im} \oint\left\langle u(\mathbf{R}) \mid \nabla_{\mathbf{R}} u(\mathbf{R})\right\rangle \cdot d \mathbf{R} .
$$

Since the Brillouin zone of a 1D periodic system can be regarded as a closed curve, Equation (A6) can be rewritten as

$$
\begin{aligned}
\Theta_{n}^{Z a k} & =-\operatorname{Im} \oint\left\langle u_{n}(q) \mid \partial_{q} u_{n}(q)\right\rangle \cdot d q \\
& =-\operatorname{Im} \int_{-\pi / \ell}^{\pi / \ell}\left\langle u_{n}(q) \mid \partial_{q} u_{n}(q)\right\rangle \cdot d q
\end{aligned}
$$

where $n$ is the band index, $\ell$ is the lattice constant and $|u(q)\rangle$ is Bloch wave function, respectively. If Zak connection evolution for the $n$th band is smooth in $q$ space, the corresponding Zak phase must vanish. However, if Zak connection evolution experiences a singularity point at $q_{0}$, Zak phase is then given by

$$
\Theta_{n}^{Z a k}=-\operatorname{Im} \lim _{\delta q \rightarrow 0}\left\{\left(\int_{-\pi / \ell}^{q_{0}-\delta q} d q+\int_{q_{0}+\delta q}^{\pi / \ell} d q\right)\left\langle u_{n, q} \mid \partial_{q} u_{n, q}\right\rangle+\int_{q_{0}-\delta q}^{q_{0}+\delta q} d q\left\langle u_{n, q} \mid \partial_{q} u_{n, q}\right\rangle\right\}
$$

As $\delta q \rightarrow 0$, the states locate at $q_{0}+\delta q$ and $q_{0}-\delta q$ are two neighboring states. As a result, we can apply Equation (A4) to the third integration term in (A9), which reads

$$
\Theta_{n}^{Z a k}=-\operatorname{Im} \lim _{\delta q \rightarrow 0}\left\{\left(\int_{-\pi / \ell}^{q_{0}-\delta q} d q+\int_{q_{0}+\delta q}^{\pi / \ell} d q\right)\left\langle u_{n, q} \mid \partial_{q} u_{n, q}\right\rangle+\ln \left\langle u_{n, q_{0}+\delta q} \mid u_{n, q_{0}-\delta q}\right\rangle\right\}
$$

According to the gauge transformation, the state around the singularity point satisfies

$$
\left|u_{n, q_{0}+}\right\rangle=-\left|u_{n, q_{0}-}\right\rangle
$$

Finally, substituting Equation (A11) into Equation (A10), Zak phase of the $n$th band is obtained by

$$
\begin{aligned}
\Theta_{n}^{Z a k} & =-\operatorname{Im} \lim _{\delta q \rightarrow 0}\left\{\left(\int_{-\pi / \ell}^{q_{0}-\delta q} d q+\int_{q_{0}+\delta q}^{\pi / \ell} d q\right)\left\langle u_{n, q} \mid \partial_{q} u_{n, q}\right\rangle+\ln \left(-\left\langle u_{n, q_{0}+\delta q} \mid u_{n, q_{0}+\delta q}\right\rangle\right)\right\} \\
& =-\operatorname{Im}[0+\ln (-1)] \\
& =\pi
\end{aligned}
$$




\section{References}

1. Bernevig, B.A.; Hughes, T.L. Topological Insulators and Topological Superconductors; Princeton University Press: Princeton, NJ, USA, 2013.

2. Atala, M.; Aidelsburger, M.; Barreiro, J.T.; Abanin, D.; Kitagawa, T.; Demler, E.; Bloch, I. Direct measurement of the Zak phase in topological Bloch bands. Nat. Phys. 2013, 9, 795-800. [CrossRef]

3. Raghu, S.; Haldane, F.D.M. Analogs of quantum-Hall-effect edge states in photonic crystals. Phys. Rev. A 2008, 78, 033834. [CrossRef]

4. Büttiker, M. Absence of backscattering in the quantum Hall effect in multiprobe conductors. Phys. Rev. B 1988, 38, 9375-9389. [CrossRef]

5. Halperin, B.I. Quantized Hall conductance, current-carrying edge states, and the existence of extended states in a two-dimensional disordered potential. Phys. Rev. B 1982, 25, 2185-2190. [CrossRef]

6. Kane, C.L.; Mele, E.J. Quantum spin Hall effect in graphene. Phys. Rev. Lett. 2005, 95, 226801. [CrossRef]

7. Kane, C.L.; Mele, E.J. Z2 topological order and the quantum spin Hall effect. Phys. Rev. Lett. 2005, 95, 146802. [CrossRef]

8. Un, I.W.; Yen, T.J. Interface States and Interface-Bulk Correspondence of One-dimensional Hyperbolic Metamaterials. Sci. Rep. 2017, 7, 43392. [CrossRef]

9. Gao, W.; Lawrence, M.; Yang, B.; Liu, F.; Fang, F.; Beri, B.; Li, J.; Zhang, S. Topological photonic phase in chiral hyperbolic metamaterials. Phys. Rev. Lett. 2015, 114, 037402. [CrossRef]

10. Ryu, S.; Hatsugai, Y. Topological origin of zero-energy edge states in particle-hole symmetric systems. Phys. Rev. Lett. 2002, 89, 077002. [CrossRef]

11. Bernevig, B.A.; Hughes, T.L.; Zhang, S.-C. Quantum Spin Hall Effect and Topological Phase Transition in HgTe Quantum Wells. Science 2006, 314, 1757. [CrossRef]

12. Haldane, F.D. Model for a quantum Hall effect without Landau levels: Condensed-matter realization of the "parity anomaly". Phys. Rev. Lett. 1988, 61, 2015-2018. [CrossRef] [PubMed]

13. Goldman, N.; Juzeliunas, G.; Ohberg, P.; Spielman, I.B. Light-induced gauge fields for ultracold atoms. Rep. Prog. Phys. 2014, 77, 126401. [CrossRef] [PubMed]

14. Cooper, N.R. Rapidly rotating atomic gases. Adv. Phys. 2008, 57, 539-616. [CrossRef]

15. Kohmoto, M. Topological Invariant and the Quantization of the Hall Conductance. Ann. Phys. 1985, 160, 343-354. [CrossRef]

16. Thouless, D.J.; Kohmoto, M.; Nightingale, M.P.; den Nijs, M. Quantized Hall Conductance in a Two-Dimensional Periodic Potential. Phys. Rev. Lett. 1982, 49, 405-408. [CrossRef]

17. Asbóth, J.K.; Oroszlány, L.; Pályi, A. A Short Course on Topological Insulators; Springer: Heidelberg, Germany, 2016.

18. Su, W.P.; Schrieffer, J.R.; Heeger, A.J. Solitons in Polyacetylene. Phys. Rev. Lett. 1979, 42, 1698-1701. [CrossRef]

19. Zak, J. Berry's Phase for Energy Bands in Solids. Phys. Rev. Lett. 1989, 62, 2747. [CrossRef]

20. Xiao, M.; Ma, G.; Yang, Z.; Sheng, P.; Zhang, Z.Q.; Chan, C.T. Geometric phase and band inversion in periodic acoustic systems. Nat. Phys. 2015, 11, 240-244. [CrossRef]

21. Xiao, M.; Zhang, Z.Q.; Chan, C.T. Surface Impedance and Bulk Band Geometric Phases in One-Dimensional Systems. Phy. Rev. X 2014, 4, 021017. [CrossRef]

22. Li, Y.; Sun, Y.; Zhu, W.; Guo, Z.; Jiang, J.; Kariyado, T.; Chen, H.; Hu, X. Topological LC-circuits based on microstrips and observation of electromagnetic modes with orbital angular momentum. Nat. Commun. 2018, 9, 4598. [CrossRef]

23. Albert, V.V.; Glazman, L.I.; Jiang, L. Topological properties of linear circuit lattices. Phys. Rev. Lett. 2015, 114, 173902. [CrossRef] [PubMed]

24. Mei, F.; You, J.-B.; Nie, W.; Fazio, R.; Zhu, S.-L.; Kwek, L.C. Simulation and detection of photonic Chern insulators in a one-dimensional circuit-QED lattice. Phys. Rev. A 2015, 92, 041805. [CrossRef]

25. Rechtsman, M.C.; Zeuner, J.M.; Plotnik, Y.; Lumer, Y.; Podolsky, D.; Dreisow, F.; Nolte, S.; Segev, M.; Szameit, A. Photonic Floquet topological insulators. Nature 2013, 496, 196-200. [CrossRef] [PubMed]

26. Haldane, F.D.; Raghu, S. Possible realization of directional optical waveguides in photonic crystals with broken time-reversal symmetry. Phys. Rev. Lett. 2008, 100, 013904. [CrossRef] [PubMed]

27. Wang, Z.; Chong, Y.; Joannopoulos, J.D.; Soljacic, M. Observation of unidirectional backscattering-immune topological electromagnetic states. Nature 2009, 461, 772-775. [CrossRef] [PubMed] 
28. Liu, S.; Gao, W.; Zhang, Q.; Ma, S.; Zhang, L.; Liu, C.; Xiang, Y.J.; Cui, T.J.; Zhang, S. Topologically Protected Edge State in Two-Dimensional Su-Schrieffer-Heeger Circuit. Research 2019, 2019, 1-8.

29. Cabot, A.; Galve, F.; Zambrini, R. Dynamical and quantum effects of collective dissipation in optomechanical systems. New J. Phys. 2017, 19, 113007. [CrossRef]

30. Deymier, P.A.; Runge, K.; Vasseur, J.O. Geometric phase and topology of elastic oscillations and vibrations in model systems: Harmonic oscillator and superlattice. AIP Adv. 2016, 6, 121801. [CrossRef]

31. Yin, J.; Ruzzene, M.; Wen, J.; Yu, D.; Cai, L.; Yue, L. Band transition and topological interface modes in 1D elastic phononic crystals. Sci. Rep. 2018, 8, 6806. [CrossRef] [PubMed]

32. Yariv, A.; Yeh, P. Optical Waves in Crystals; Wiley: New York, NY, USA, 1948.

33. Choi, K.H.; Ling, C.W.; Lee, K.F.; Tsang, Y.H.; Fung, K.H. Simultaneous multi-frequency topological edge modes between one-dimensional photonic crystals. Opt. Lett. 2016, 41, 1644-1647. [CrossRef]

34. Prodan, E.; Dobiszewski, K.; Kanwal, A.; Palmieri, J.; Prodan, C. Dynamical Majorana edge modes in a broad class of topological mechanical systems. Nat. Commun. 2017, 8, 14587. [CrossRef] [PubMed]

(C) 2019 by the authors. Licensee MDPI, Basel, Switzerland. This article is an open access article distributed under the terms and conditions of the Creative Commons Attribution (CC BY) license (http://creativecommons.org/licenses/by/4.0/). 